\title{
The efficacy and safety of whole-body electromyostimulation in applying to human body: based from graded exercise test
}

\author{
Yong-Seok Jee ${ }^{1,2 * *}$ \\ ${ }^{1}$ Research Institute of Sports and Industry Science, Hanseo University, Seosan, Korea \\ 2Department of Physical Activity Design, Hanseo University, Seosan, Korea
}

Recently, whole body-electromyostimulation (WB-EMS) has upgraded its functions and capabilities and has overcome limitations and inconveniences from past systems. Although the efficacy and safety of EMS have been examined in some studies, specific guidelines for applying WB-EMS are lacking. To determine the efficacy and safety of applying it in healthy men to improve cardiopulmonary and psychophysiological variables when applying WB-EMS. Sixty-four participants were randomly grouped into control group (without electrical stimuli) or WBEMS group after a 6 -week baseline period. The control group $(n=33$; female. 15; male, 18) wore the WB-EMS suit as much as the WB-EMS group ( $n=31$; female, 15; male, 16). There were no abnormal changes in the cardiopulmonary variables (heart rate, systolic blood pressure [SBP], diastolic blood pressure, and oxygen uptake) during or after the graded exercise test (GXT) in both groups. There was a significant decrease in SBP and an increase of oxygen uptake from stages 3 to 5 of the GXT in the WB-EMS group. The psychophysiological factors for a WB-EMS group, which consisted of soreness, anxiety, fatigability, and sleeplessness were significantly decreased after the experiment. The application of WB-EMS in healthy young men did not negatively affect the cardiopulmonary and psychophysiological factors. Rather, the application of WB-EMS improved SBP and oxygen uptake in submaximal and maximal stages of GXT. This study also confirmed that 6 weeks of WB-EMS training can improve psychophysiological factors.

Keywords: Whole-body electromyostimulation, Heart rate, Systolic blood pressure, Oxygen uptake, Graded exercise test

\section{INTRODUCTION}

Electromyostimulation (EMS) has been used for physical therapy practice as a method to rehabilitate muscles after an injury or surgery. In the 1960s, EMS was often used to prevent the atrophy that occurs when skeletal muscle is denervated. With advances in EMS technology, its use became increasingly popular for treating patients who sustained central nervous system impairment secondary to brain injury. Since the 1980s, researchers have developed units with an improved ability to modulate a variety of electrical wave forms resulting in an electrical current that can be effectively used to stimulate innervated muscles (Porcari et al., 2002). It has been commonly used to strengthen the extremities of patients who have had orthopedic surgery. The use of EMS can also prevent people from injuries, especially for those inclined to injuries in the lower back, knees, shoulders, and muscles. EMS is very gentle on the joints and reduces the risk of injury due to the absence of weights (Kemmler et al., 2010).

During EMS, impulses are transmitted through electrodes on the skin close to dermis tissue for stimulation. These impulses cause involuntary contractions of the muscles and thereby recruit fast-twitch fibers (Lexell et al., 1988). The major difference between the mechanisms of involuntary muscle contraction and voluntary muscle contraction induced by EMS is in muscle fiber mobilization. In order to mobilize muscle fiber, there must be a level of stimulation that can be activated. In the case of spontaneous muscle contraction, a small motor unit with low threshold is activated first. In the case of muscle contraction due to EMS, the mo-

\footnotetext{
${ }^{*}$ Corresponding author: Yong-Seok Jee (iD https://orcid.org/0000-0001-6797-0843 Research Institute of Sports and Industry Science, Hanseo University, 46 Hanseo 1-ro, Haemi-myeon, Seosan 31962, Korea Tel: +82-41-660-1028, Fax: +82-41-660-1088, E-mail: jeeys@hanseo.ac.kr Received: January 2, 2018 / Accepted: January 30, 2018
}

This is an Open Access article distributed under the terms of the Creative Commons Attribution Non-Commercial License (http://creativecommons.org/licenses/by-nc/4.0/) which permits unrestricted non-commercial use, distribution, and reproduction in any medium, provided the original work is properly cited. 
tor unit under the control of the larger nerve is activated, and muscle fibers with high threshold are easily mobilized (Enoka, 2002), resulting in positive effects on muscle strength (Gondin et al., 2005). Gregory and Bickel (2005) also suggested that voluntary contraction may result in selective motor unit mobilization from slow- to fast-muscle fibers, but nonselective contraction occurs when both fibers are mobilized simultaneously during involuntary contraction through EMS. Variables that affect the physiological changes of the nerves and muscles in EMS application include frequency, intensity or current amplitude, pulse characteristics, current/rest cycle, pulse ramping, electrode type, material, size, and placement (Cameron, 2012).

Many studies applied EMS locally with single electrodes to defined muscle groups (Porcari et al., 2002). With further technical developments, EMS progressed from a local stimulation to a whole body-EMS (WB-EMS) method where several muscle groups were targeted simultaneously through an electrode belt and vest system (i.e., Miracle Suit, Seoul, Korea). More recently, Miracle Suit has upgraded its functions and capabilities and has overcome limitations and inconveniences from past systems. For example, the cumbersome process of spraying water or wearing wet clothes to allow electrical currents to pass through the body has been replaced by inserting a silicone conductive pad into the garment. In addition, wires connecting the electrodes to the EMS machine have been replaced with wireless sensors via Bluetooth technology to enable a wider range of activity that can be adjusted within $40 \mathrm{~m}$. This advanced WB-EMS system allows for more comfort, ease of use, and faster results in rehabilitating patients with musculoskeletal diseases as well as improving body composition and muscle function in normal people as shown in previous studies (Kemmler and von Stengel, 2013; Kemmler et al., 2010; Kemmler et al., 2016a; von Stengel et al., 2015).

As is often the case, the equipment that is used in clinical practice is not without its drawbacks, as reported by Malnick et al. (2016) regarding the potential risks of WB-EMS. In general, WB-EMS undeniably features factors known to be associated with resistance exercise-induced muscle damage and pronounced muscle soreness, which can lead to exertional rhabdomyolysis (Finsterer and Stöllberger, 2015; Kästner et al., 2015; Koch et al., 2014). However, to avoid WB-EMS-related side effects, guidelines for efficacy and safety were established, which included the control of electrical strength, consideration of subject health, and the training of instructors (Kemmler et al., 2016b). Recently, researchers have used the recommended WB-EMS protocol (20 min, bipolar, $85 \mathrm{~Hz}, 350 \mu \mathrm{sec}$, rectangular, $6 \mathrm{sec}$ of current, $4 \mathrm{sec}$ of rest) for healthy men (Filipovic et al., 2011; Kemmler and von Stengel, 2013; Kemmler et al., 2010; Kemmler et al., 2016b; Lam and Qin, 2008). It was reported that using the WB-EMS protocols in their studies can prevent rhabdomyolysis and corresponding renal, hepatic, and cardiac problems.

However, although the efficacy and safety of WB-EMS have been examined in some studies, specific guidelines for investigating the changes in cardiopulmonary function are lacking. In particular, there are no reports on the effects of WB-EMS on psychophysiological factors. Therefore, this study investigated the changes in cardiopulmonary and psychophysiological factors when applying the recommended range of WB-EMS which was shown to be safe and effective.

\section{MATERIALS AND METHODS}

\section{Subjects}

The subjects were aged between 20 and 25 from Hanseo University located in Seosan, South Korea. Prior to the study, the participants received detailed explanations regarding all the procedures of this study and were then asked to complete questionnaires. Inclusion criteria included having no abnormalities in medical tests from January to December 2017 and confirming a good medical history without any injuries or chest pain during the past year. All of the subjects who took part in this study were healthy collegiate students who did not exercise regularly for a duration of 6 months. Additionally, the subjects were also included if they had not taken any treatment for weight loss or any medication known to affect body composition, had not undergone any major surgery during the 1 year prior to the start of the study, and did not have any internal metallic materials such as a pacemaker. Exclusion criteria included any history of coronary arterial disease or cerebrovascular disease, impairment of a major organ system, severe lung disease, severe cerebral trauma, uncontrolled hypertension, cancer, and psychiatric diseases such as eating disorders. Participants with musculoskeletal dysfunction or pain were also excluded from this study.

Of the 71 participants who completed the survey, 3 subjects were disqualified. One of them took part in an exercise program over 1 year, one of them refused to participate, and the other had personal reasons. The remaining 68 participants were randomly allocated to each group. As shown in Fig. 1, the control group consisted of 15 female and 18 male subjects for this study after one participant was disqualified due to a failure of receiving assessments and discontinuing the study. The WB-EMS group con- 


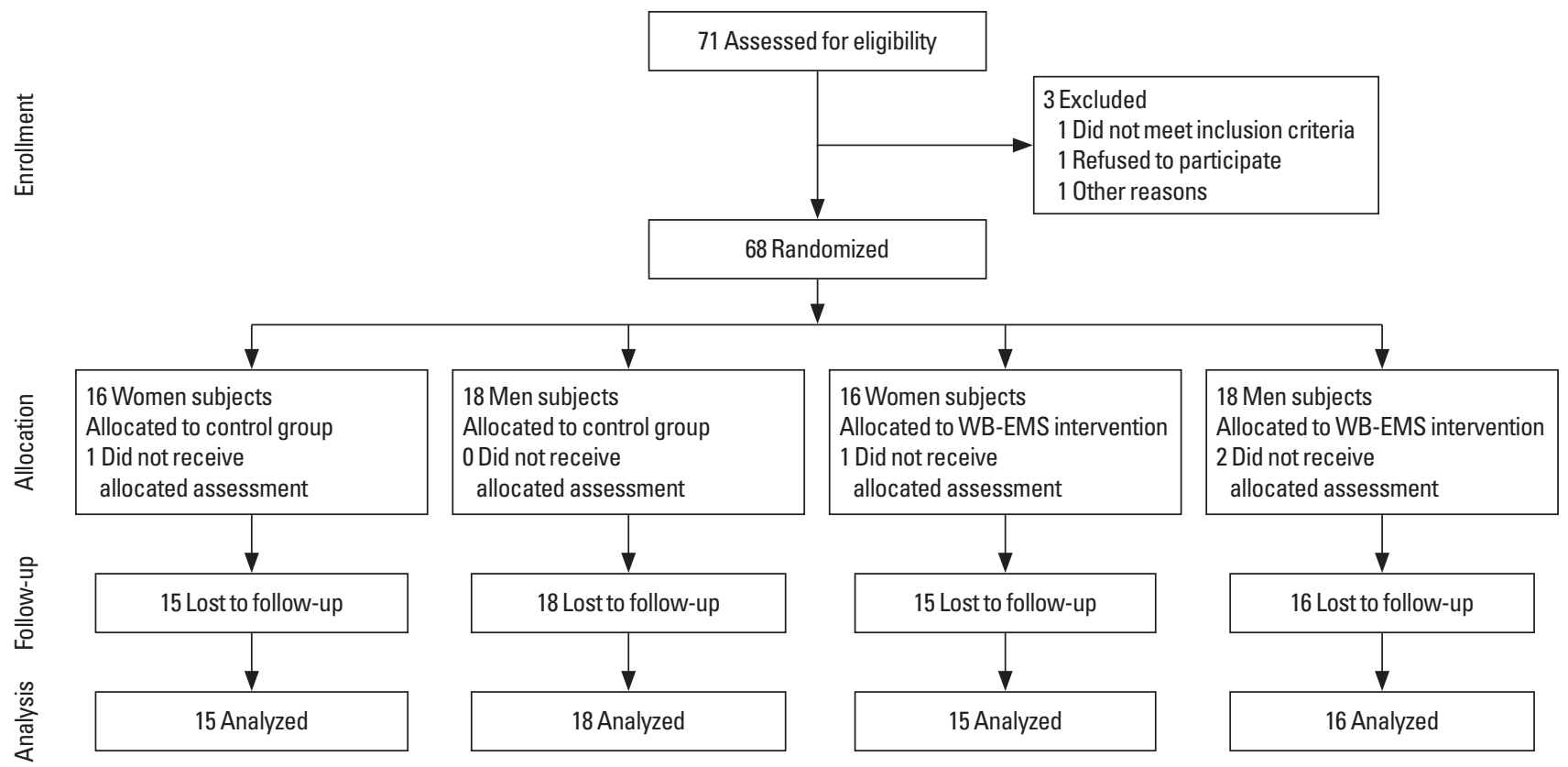

Fig. 1. Flow diagram participants (assessed for eligibility; $n=71$ ).

sisted of 15 female and 16 male subjects after three participants were disqualified due to a failure of receiving assessments. The mean (standard deviation, SD) age of the subjects was 21.91 (1.20) years and they were divided into two groups: control group $(\mathrm{n}=33)$ and WB-EMS group $(\mathrm{n}=31)$. Participants were assigned to these groups using random number tables and assigned identification numbers upon recruitment. No participant, investigator, or other staff members interacting with the participants were aware of the group assignments for the duration of the trial. Although the participants of the control group wore the WB-EMS suit as much as the experiment group, they did not receive any electrical stimuli. The program lasted 6 weeks, similar to the duration of previous studies (Filipovic et al., 2011; Gondin et al., 2011; Hortobágyi and Maffiuletti, 2011; Kemmler and von Stengel, 2013; Kemmler et al., 2010; Kemmler et al., 2016b; Lam and Qin, 2008). Complete subject characteristics are presented in Table 1.

\section{Experimental design}

This double-blind, randomized, controlled trial was conducted in a research center at Hanseo University from July to September, 2017. The first assessment was conducted in Seoul at Song-Do Hospital in the beginning of July, 2017 and the second assessment was conducted in the same hospital in late September. The study was approved by the Institutional Review Board at Hanseo
Table 1. Physical characteristics of the subjects

\begin{tabular}{lcccc}
\hline Characteristic & $\begin{array}{c}\text { Control group } \\
(\mathrm{n}=33)\end{array}$ & $\begin{array}{c}\text { WB-EMS group } \\
(\mathrm{n}=31)\end{array}$ & \multicolumn{2}{c}{ Kruskal-Wallis test } \\
\cline { 5 - 5 } & & & $X^{2}$ & $P$-value \\
\hline Age $(\mathrm{yr})$ & $21.34 \pm 0.93$ & $22.21 \pm 1.23$ & 6.889 & 0.076 \\
Height $(\mathrm{cm})$ & $171.67 \pm 2.89$ & $173.85 \pm 4.17$ & 5.786 & 0.123 \\
Weight $(\mathrm{kg})$ & $71.37 \pm 5.71$ & $75.04 \pm 4.33$ & 1.510 & 0.680 \\
SMM $(\mathrm{kg})$ & $32.93 \pm 0.95$ & $36.65 \pm 5.30$ & 1.621 & 0.655 \\
BFM $(\mathrm{kg})$ & $13.60 \pm 4.41$ & $16.30 \pm 3.13$ & 1.333 & 0.721 \\
BMI $\left(\mathrm{kg} / \mathrm{m}^{2}\right)$ & $24.27 \pm 2.60$ & $25.30 \pm 2.19$ & 0.675 & 0.879 \\
WHR & $0.86 \pm 0.02$ & $0.87 \pm 0.03$ & 1.038 & 0.792 \\
BMR $(\mathrm{kcal})$ & $1,617.33 \pm 33.26$ & $1,662.50 \pm 191.75$ & 1.648 & 0.249 \\
\hline
\end{tabular}

Values are presented as mean \pm standard deviation.

WB-EMS, whole body-electromyostimulation; SMM, skeletal muscle mass; BFM, body fat mass; BMI, body mass index; WHR, waist-hip ratio; BMR, basal metabolic rate.

University and Sahm Yook University (2-1040781-AB-N-012017083HR). All subjects were recruited through advertisements and a written informed consent was obtained before enrollment. First, all of the subjects arrived at the research center to sign an informed consent form and to complete a self-report questionnaire about their health status included in the physical examination. After this procedure, each subject participated in the experiment conducted by an expert. The measurement and experimental rooms had the same characteristics with respect to temperature $\left(25^{\circ} \mathrm{C}-26^{\circ} \mathrm{C}\right)$ and humidity $(50 \%-60 \%)$. 


\section{Measurement methods}

\section{Graded exercise test}

The graded exercise test (GXT) is a diagnostic test used to rule out coronary artery disease and/or abnormal rhythms and to evaluate exercise capacity in the hospital. The cardiovascular functions before and after the application of WB-EMS were analyzed by an EKG tester (Q-4500) including a treadmill (Q65-90), an automatic sphygmomanometer (M-412), and a GAS tester (QMC4200). The Bruce protocol was applied in consideration of the healthy young women and men. In the Bruce protocol, the starting point (stage 1 ) was $1.7 \mathrm{mph}$ at a $10 \%$ grade (5 metabolic equivalents [METs]). Stage 2 was $2.5 \mathrm{mph}$ at a $12 \%$ grade (7 METs), stage 3 was $3.4 \mathrm{mph}$ at a $14 \%$ grade (9 METs), stage 4 was $4.2 \mathrm{mph}$ at a $16 \%$ grade (11 METs), and subsequent stages followed similar increases in speed and grade. This protocol included 3-min periods to achieve a steady state before workload was increased. All subjects were instructed to continue to walk or jog until reaching an all-out level which is their maximal ratings perceived exertion (RPE). During and after walking or running on the treadmill for as long as possible, the subjects were instructed to describe their symptoms as follows: chest pain, shortness of breath, dizziness, and leg pain. During the test, subjects were asked to express their level of exercise intensity on the RPE scale. The test was terminated if the following symptoms occurred: (a) drop in systolic blood pressure (SBP) of more than $10 \mathrm{mmHg}$ from baseline, despite an increase in workload, when accompanied by other evidence of ischemia, (b) moderate-to-severe angina, (c) increase in nervous system symptoms (e.g., ataxia, dizziness, near-syncope), (d) signs of poor perfusion (cyanosis), (e) technical difficulties in monitoring electrocardiographic tracings or SBP, (f) subject's desire to stop, (g) sustained ventricular tachycardia, and (h) ST elevation ( $>1 \mathrm{~mm}$ ) in leads without diagnostic $\mathrm{Q}$ waves (other than V1 or aVR) (American College of Sports Medicine, 2013). The variables of this study were limited to heart rate (HR), SBP, diastolic blood pressure (DBP), and oxygen uptake $\left(\mathrm{VO}_{2}\right)$ calculated by body weight every minute at each test stage.

\section{Psychophysiological factors}

An important emphasis was placed on how the subjects reacted to the WB-EMS trial in terms of psychophysiological factors (soreness, anxiety, fatigue, and sleeplessness). A visual analogue scale (VAS) was used to measure psychophysiological factors. All of the subjects in the experimental group were asked to rate how they felt as they received WB-EMS training using a bipolar rating scale, which is a bar-shaped box with a height of $5 \mathrm{~cm}$ and a length of $10 \mathrm{~cm}$. The scale ranged from no soreness (close to "0") to severe soreness (close to "10"). After the subjects marked within the box, a transparent paper with score indicators was placed on top of the boxes to obtain a numerical score. The scale for measuring the levels of anxiety, fatigue, and sleeplessness were similar to the soreness scale. The subjects were evaluated by a professional psychologist at the beginning and end of the study. The reliability of the questionnaire was measured by calculating Cronbach $\alpha$, representing internal consistency. The Cronbach $\alpha$ of soreness, anxiety, fatigue, and sleeplessness scales were from 0.69 to 0.81 .

\section{WB-EMS administration}

Participants were given WB-EMS suits made by Miracle (Seoul, Korea) according to their size. Their garments were composed of a silicone conductive pad and wireless materials. The electrical strength of the suit was controlled by an i-pad or cellular phone via Bluetooth. WB-EMS equipment used in this study enabled the simultaneous activation of 8 muscle groups (both upper legs, both upper arms, buttocks, abdomen, chest, lower back, upper back, and latissimus dorsi) with selectable intensities for each region (Fig. 2).

Based on available literature, the stimulation-frequency was selected at $85 \mathrm{~Hz}$, the impulse-width at $350 \mu \mathrm{sec}$, the impulse-rise as rectangular application, and variable electrostimulation intensity relative to the maximal peak voltage $(160 \mathrm{~V})$. Impulse duration was 6 sec with a 4-sec break between impulses (Filipovic et al., 2011; Kemmler and von Stengel, 2013; Kemmler et al., 2010; Kemmler et al., 2016a; Lam and Qin, 2008). For each group, a qualified instructor conducted 20-min WB-EMS sessions 3 times a week on two nonconsecutive days to allow a rest interval of 48 hr between each session. In order to generate the effects of a diverse range of motions, ten types of isometric movements composed of Crunches, Bridges, Leg raises, Side planks on right and left sides, Superman back, Front planks, Lunges on right and left sides, and Squats were performed during the impulse phase as per the instructor's direction as shown in Fig. 2. This study estimated one maximal toleration (1-MT) to the stimulus current on maximum peak voltage, similar to calculating the maximal voluntary contraction (MVC) or one maximal repetition (1-RM). Recently, Gondin et al. (2011) reported that the maximal toleration, expressed in this study as the corresponding incomplete muscle activation, can explain the inability to produce electrically-evoked force equal to 100\% MVC. 1-MT was determined by progressively administering electrical intensity until the subjects expressed 
1st Crunch

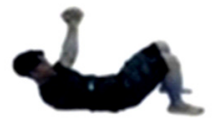

6th Superman back

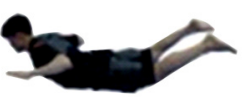

2nd Bridge

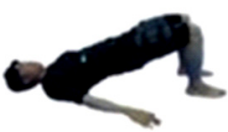

7th Front plank

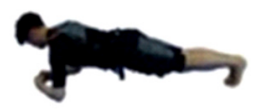

3rd Leg raise

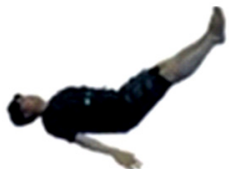

8th Lunge (right)

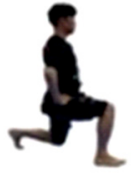

4th Side plank (right)

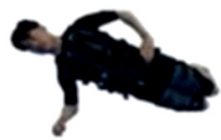

9th Lunge (left)

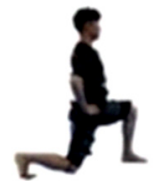

5th Side plank (left)

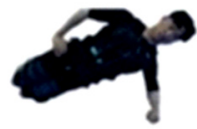

10th Squat

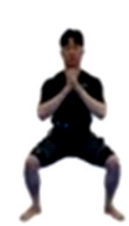

Fig. 2. Isometric exercises wore whole body-electromyostimulation (WB-EMS) suit.

their maximum tolerance (Bily et al., 2008).

American College of Sports Medicine (2013) describes exercise intensity based on the concept of 1-RM in the recommendation for resistance exercise in which $50 \%$ of 1-RM is considered light intensity, $60 \%$ moderate intensity, and $80 \%$ vigorous intensity. In this experiment, 1-MT was measured and the exercise intensity was set by using the 1-RM concept. In an orientation session before initiating the electrical stimulation exercise, each participant of the WB-EMS group stood still while wearing WB-EMS suits. Starting from $10 \%$ of 1-MT, the intensity was gradually increased according to the response of the participant, and the electric stimulation was stopped at the request of the participant when reaching an unbearable level, at which point the intensity was set to 1-MT. The participants were assigned to $0 \%$ of $1-\mathrm{MT}$ in control group and $80 \%$ of 1 -MT $(125.21 \pm 7.82 \mathrm{~V})$ in WB-EMS group, respectively.

\section{Statistical analyses}

The sample sizes in this study were not adequate since a statistical power greater than $90 \%$ was achieved for the standardized differences between time greater than $5 \%$ at a probability of type-I error $<0.05$. All data are reported as mean (SD). The Kolmogorov-Smirnov test was used to determine the normality of distribution for the examined variables. In order to examine the differences of the GXT variables between control group and WBEMS group, nonparametric tests (two-independent samples tests Mann-Whitney $U$-test) were completed. Meanwhile, in order to examine the differences of psychophysiological factors between times of WB-EMS group, nonparametric tests (two-related samples tests - Wilcoxon signed-ranks test) were performed. The significance level for all analyses was set a priori at $P<0.05$. The
IBM SPSS ver. 18.0 (IBM Co., Armonk, NY, USA) was used to analyze the statistics for this study.

\section{RESULTS}

\section{Effect of WB-EMS on HR during GXT}

Around $34.37 \%$ of the subjects (control group, $n=8$; WB-EMS group, $n=14$ ) were performed until the stage 5 of GXT in this study. The data from HR for both groups in GXT were analyzed for differences after the experiment. As shown in Table 2, the HRs of control group and WB-EMS group were changed normally according to their GXT stages. There were significant differences of HRs between resting and stage 1 for both groups only at the early stage of the GXT, but there were no significant differences in subsequent test stages and the recovery stage. These results revealed that the application of WB-EMS did not negatively affect HR.

\section{Effect of WB-EMS on blood pressures during GXT}

The data from SBP for both groups in GXT were analyzed for differences after the experiment. As shown in Table 3, the SBPs of control group and WB-EMS group changed normally according to their GXT stages. There were significant differences of SBPs from stages 3 to 5 for both groups, but there were no significant differences in subsequent test stages and the recovery stage. These results revealed that the application of WB-EMS did not negatively affect SBP. These results were similar in DBP as shown in Table 4.

\section{Effect of WB-EMS on $\mathrm{VO}_{2}$ during GXT}

The $\mathrm{VO}_{2}$ data for both groups in GXT were analyzed for differences after the experiment. As shown in Table 5, the $\mathrm{VO}_{2}$ levels of 
Table 2. Changes and differences of heart rates between groups in stages of graded exercise test

\begin{tabular}{lccccccc}
\hline Group & Resting & Stage 1 & Stage 2 & Stage 3 & Stage 4 & Stage 5 & Recovery \\
\hline Control & $78.58 \pm 10.59$ & $117.88 \pm 24.50$ & $137.92 \pm 22.81$ & $174.48 \pm 16.99$ & $186.13 \pm 11.26$ & $193.46 \pm 8.51$ & $80.06 \pm 11.26$ \\
WB-EMS & $73.20 \pm 8.22$ & $121.47 \pm 18.70$ & $138.05 \pm 21.57$ & $170.77 \pm 17.71$ & $184.13 \pm 8.41$ & $194.15 \pm 7.66$ & $77.00 \pm 11.00$ \\
$Z^{*}$ & -5.454 & -2.625 & -0.532 & -0.714 & -1.335 & -0.793 & -0.682 \\
$P$-value & 0.002 & 0.009 & 0.594 & 0.476 & 0.182 & 0.428 & 0.482 \\
\hline
\end{tabular}

Values are presented as mean \pm standard deviation. WB-EMS group received electrical stimuli as an experiment group.

WB-EMS, whole body-electromyostimulation.

${ }^{*}$ Represents the statistical analysis method Mann-Whitney U-test.

Table 3. Changes of systolic blood pressures in times and groups

\begin{tabular}{lccccccc}
\hline Group & Resting & Stage 1 & Stage 2 & Stage 3 & Stage 4 & Stage 5 & Recovery \\
\hline Control & $123.20 \pm 6.64$ & $161.19 \pm 15.65$ & $181.78 \pm 20.33$ & $198.13 \pm 20.64$ & $218.98 \pm 23.04$ & $238.00 \pm 10.78$ & $119.20 \pm 16.64$ \\
WB-EMS & $122.23 \pm 4.41$ & $162.08 \pm 13.52$ & $182.34 \pm 19.21$ & $196.41 \pm 20.82$ & $214.64 \pm 21.05$ & $231.74 \pm 13.22$ & $117.23 \pm 12.41$ \\
$Z^{*}$ & -1.915 & -1.753 & -0.398 & -2.793 & -5.544 & -5.035 & -0.969 \\
$P$-value & 0.056 & 0.080 & 0.690 & 0.005 & 0.001 & 0.001 & 0.065
\end{tabular}

Values are presented as mean \pm standard deviation. WB-EMS group received electrical stimuli as an experiment group.

WB-EMS, whole body-electromyostimulation.

${ }^{*}$ Represents the statistical analysis method Mann-Whitney U-test.

Table 4. Changes of diastolic blood pressures in times and groups

\begin{tabular}{lccccccc}
\hline Group & Resting & Stage 1 & Stage 2 & Stage 3 & Stage 4 & Stage 5 & Recovery \\
\hline Control & $82.75 \pm 7.09$ & $82.33 \pm 8.99$ & $78.86 \pm 9.58$ & $81.69 \pm 8.14$ & $82.66 \pm 10.38$ & $79.26 \pm 8.57$ & $77.75 \pm 11.09$ \\
WB-EMS & $80.95 \pm 5.64$ & $78.56 \pm 7.84$ & $80.95 \pm 5.64$ & $80.89 \pm 5.07$ & $80.64 \pm 4.66$ & $78.21 \pm 7.47$ & $76.95 \pm 9.64$ \\
$Z^{*}$ & -2.990 & -3.199 & -0.953 & -0.482 & -1.153 & -2.798 & -0.487 \\
$P$-value & 0.003 & 0.001 & 0.341 & 0.629 & 0.249 & 0.005 & 0.697 \\
\hline
\end{tabular}

Values are presented as mean \pm standard deviation. WB-EMS group received electrical stimuli as an experiment group.

WB-EMS, whole body-electromyostimulation.

${ }^{*}$ Represents the statistical analysis method Mann-Whitney U-test.

Table 5. Changes of oxygen uptake in times and groups

\begin{tabular}{lccccccc}
\hline Group & Resting & Stage 1 & Stage 2 & Stage 3 & Stage 4 & Stage 5 & Recovery \\
\hline Control & $7.15 \pm 2.06$ & $18.68 \pm 4.54$ & $28.85 \pm 5.59$ & $38.81 \pm 6.45$ & $48.49 \pm 8.98$ & $60.33 \pm 4.22$ & $8.15 \pm 6.06$ \\
WB-EMS & $7.28 \pm 1.72$ & $18.83 \pm 4.32$ & $28.99 \pm 5.19$ & $39.24 \pm 5.91$ & $49.85 \pm 8.83$ & $62.93 \pm 3.82$ & $9.27 \pm 7.72$ \\
$Z^{*}$ & -1.337 & -1.633 & -1.002 & -2.302 & -2.349 & -2.083 & -1.425 \\
$P$-value & 0.181 & 0.103 & 0.316 & 0.021 & 0.019 & 0.037 & 0.169 \\
\hline
\end{tabular}

Values are presented as mean \pm standard deviation. WB-EMS group received electrical stimuli as an experiment group.

WB-EMS, whole body-electromyostimulation.

${ }^{*}$ Represents the statistical analysis method Mann-Whitney U-test.

control group and WB-EMS group changed normally according to their GXT stages. There were significant differences in the $\mathrm{VO}_{2}$ levels from stages 3 to 5 for both groups, but there were no significant differences in the subsequent test stages and the recovery stage. These results revealed that the application of WB-EMS did not negatively affect $\mathrm{VO}_{2}$.

\section{Effect of WB-EMS on psychophysiological factors during GXT}

The data from psychophysiological factors for the WB-EMS group were analyzed for differences between pre- and postvalues after the experiment. As shown in Fig. 3, although the soreness level before the experiment was $7.70 \pm 1.15$, the level after the experiment was significantly decreased by $4.16 \pm 1.20(Z=-6.726$, $P=0.001$ ). The anxiety level before the experiment was $6.50 \pm 1.10$, 


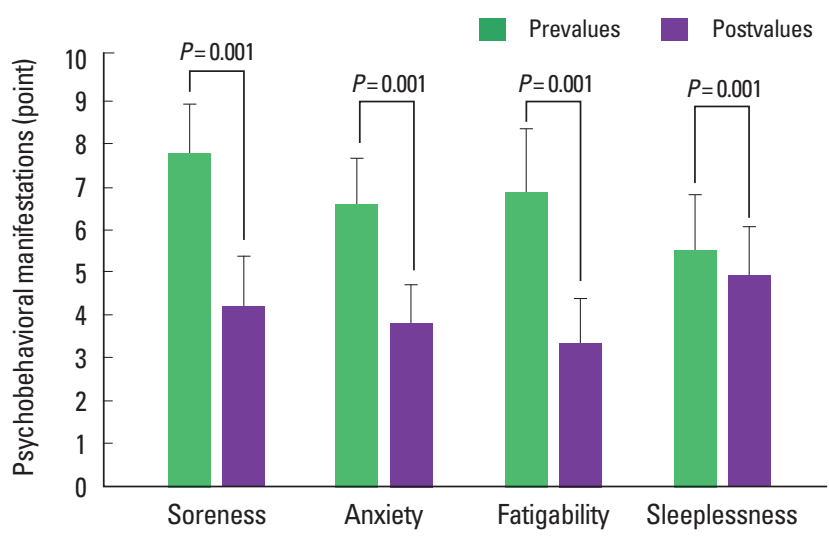

Fig. 3. Differences of psychophysiological manifestations between pre- and postvalues in whole body-electromyostimulation group. All data was analyzed by Wilcoxon signed-rank test.

but it was significantly decreased by $3.75 \pm 0.91(Z=-7.023$, $P=0.001)$ after the experiment. Although the level of fatigue and sleeplessness before the experiment were $6.81 \pm 1.46$ and $5.44 \pm 1.31$ respectively, the levels after the experiment were significantly decreased by $3.33 \pm 1.01(Z=-6.927, P=0.001)$ and $4.88 \pm 1.13$ $(Z=-4.697, P=0.001)$, respectively. These results suggest that the application of WB-EMS did not negatively affect psychophysiological factors.

\section{DISCUSSION}

The major findings of this study revealed that the application of WB-EMS intensity confirmed from previous studies did not negatively affect $\mathrm{HR}$, systolic or $\mathrm{DBP}$, and $\mathrm{VO}_{2}$ in healthy young men during and after graded exercise test. Moreover, the psychophysiological factors which included soreness, anxiety, fatigue, and sleeplessness, were significantly decreased after the experiment. The WB-EMS intervention which was composed of $85 \mathrm{~Hz}$ (frequency), $350 \mu \mathrm{sec}$ (impulse-width), mean (SD) voltages $(125.21 \pm 7.82 \mathrm{~V})$ of $80 \%$ of 1-MT, and 6 vs. 4 sec (impulse vs. break duration) applied in this study was combined with isometric strengthening exercises. The WB-EMS training as a direct rectangular application was conducted $20 \mathrm{~min}$ a day, 3 times a week. Ten types of isometric exercises were performed with 12 repetitions during the impulse phase as per the instructor's directions.

The purpose of this study was to investigate whether there would be positive or negative change in cardiopulmonary and psychophysiological functions when applying the recommended specifications of WB-EMS, which were shown to be safe and ef- fective. Although some side-effects from WB-EMS has been suggested in some studies, it has been limited to rhabdomyolysis. In that regard, Kemmler et al. (2016a) reported that WB-EMS featured many factors known to be associated with muscle damage. A recent WB-EMS study applying an initial application to exhaustion to healthy novices confirmed the reported exceptionally high creatine-kinase concentrations. In other words, studies investigating the changes in cardiopulmonary or psychophysiological factors after applying WB-EMS are lacking.

In the past, researchers have used EMS with low-frequency and was primarily performed locally, passively, therapeutically, or for athletic applications (Porcari et al., 2002). The low-frequency electrical stimulation we use refers to frequencies from 1 to 1,000 $\mathrm{Hz}$, which can penetrate the human skin and subcutaneous fat. Midfrequency electrical stimulation refers to a frequency of 1,000 to $100,000 \mathrm{~Hz}$, which can penetrate the body's muscles, but can also be used to relax the muscles that are bundled, or for intracoronary blood circulation. Of these electrical frequencies, there are many advantages of using low-frequencies. For example, stimulation of acupuncture points by low-frequency stimulation can have a large effect (Enoka, 2002). Even when emitting low microcurrents to the body, it plays a role in bioelectronics that can recover injured muscle fibers, promote maximal strength gains, and reduce cellulite, which is present in overweight people (Ahmad and Hasbullah, 2015). Specifically, according to a research study (Banerjee et al., 2005), the peak $\mathrm{VO}_{2}$ increased by an average of $0.24 \pm 0.16 \mathrm{~L} / \mathrm{min}$ and walking distance increased by $36.6 \pm 19.7$ $\mathrm{m}$ after 6 weeks. It confirmed that EMS can be used in sedentary adults (range, 25-72 years) to improve physical fitness and may provide a viable alternative to more conventional forms of exercise. There were no reported side-effects from EMS muscle training, however increases of $10 \%$ were observed in peak $\mathrm{VO}_{2}$ and treadmill test durations after EMS combined exercise training. In terms of safety, the results of this study showed that WB-EMS did not cause any side effects. In other words, the HR, SBP, and DBP of the WB-EMS group in every GXT were not significantly different compared to the control group. The responses from each stage of GXT in the WB-EMS group also changed normally. Meanwhile, similar to the study results of Banerjee et al. (2005), the results of this study showed that the $\mathrm{VO}_{2}$ levels of the WBEMS group were significantly higher than those of the control group from stages 3 to 5 of the GXT. These results show that the application of WB-EMS positively affected the submaximal or maximal $\mathrm{VO}_{2}$.

The EMS during exercise training was generally well tolerated 
by subjects with a good level of compliance (Shankar et al., 1990). Similar to physical adaptation to WB-EMS, this study showed significant decline in the psychophysiological factors including soreness, anxiety, fatigue, and sleeplessness after the experiment. Shankar et al. (1990) revealed that the difference between subjects' self-reported adherence and objectively assessed evidence of adherence might vary by up to $50 \%$. Banerjee et al. (2005) also reported that although EMS with exercise is associated with and will be limited by perceived exertion on behalf of the individual undertaking the exercise, no voluntary effort is required to participate in this form of exercise. The work is created by means of EMS-induced muscle contractions. It is reasonable to expect that the improvements seen here in sedentary untrained adults could also be achieved in populations unable to engage in voluntary exercise. Similarly, Harvey et al. (1992) suggested that the physiological benefits gained from functional EMS training led to significant psychological benefits as well. Anderson et al. (2006) reported that thirty-seven sedentary healthy women participated in baseline testing on a range of anthropometric measures, body composition, and self-perception measures. Subsequently, participants were randomly assigned an 8-week program of one of three groups: walking group, walking+EMS group, or control group. When comparing the results with the control group after the experiment, both walking groups had a significant reduction in the number of anthropometric measures and improvements in self-perception measures. The improvements on both anthropometric measures and self-perception were greater for the walking+EMS group, which indicates that changes in self-perception may be affected by physiological changes. These results are similar to previous research studies suggesting that exercise enhances self-perception (Aşçi, 2003; Taylor and Fox, 2005).

Ultimately, this study suggests that a whole-body suit equipped with an electrical muscle stimulation device may improve cardiopulmonary factors and psychophysiological indications. However, this form of exercise is not likely to be suitable for all individuals since many find EMS difficult to tolerate. Although most subjects of the WB-EMS group tolerated it quite well and there were no participants who dropped out from the experiment, five subjects in the WB-EMS group expressed tightness and discomfort in the chest area. Therefore, it is preferable to cut off the electrical stimuli to the chest area or to refrain from adding the chest area to the WB-EMS suit when it is developed later. Further research is required to confirm the most effective methods of using WB-EMS suits, to identify the populations in which it is most beneficial, and to ensure a greater degree of medical safety. In spite of these areas, this study was the first to investigate the safety of WB-EMS through applying GXT and analyzing the effects of WB-EMS on psychophysiological variables.

\section{CONFLICT OF INTEREST}

No potential conflict of interest relevant to this article was reported.

\section{ACKNOWLEDGMENTS}

This research was supported by Basic Science Research Program through the National Research Foundation of Korea (NRF) funded by the Ministry of Education (NRF-2017R1D1A1B03034766).

\section{REFERENCES}

Ahmad MF, Hasbullah AH. The effects of electrical muscle stimulation (EMS) towards male skeletal muscle mass. Int J Med Health Biomed Bioeng Pharm Eng 2015;9:860-869.

American College of Sports Medicine. ACSM's Guidelines for exercise testing and prescription. 9th ed. Philadelphia (PA): Lippincott Williams \& Wilkins; 2013.

Anderson AG, Murphy MH, Murtagh E, Nevill A. An 8-week randomized controlled trial on the effects of brisk walking, and brisk walking with abdominal electrical muscle stimulation on anthropometric, body composition, and self-perception measures in sedentary adult women. Psychol Sport Exerc 2006;7:437-451.

Aşçi FH. The effects of physical fitness training on trait anxiety and physical self-concept of female university students. Psychol Sport Exerc 2003;4:255-264

Banerjee P, Clark A, Witte K, Crowe L, Caulfield B. Electrical stimulation of unloaded muscles causes cardiovascular exercise by increasing oxygen demand. Eur J Cardiovasc Prev Rehabil 2005;12:503-508.

Bily W, Trimmel L, Mödlin M, Kaider A, Kern H. Training program and additional electric muscle stimulation for patellofemoral pain syndrome: a pilot study. Arch Phys Med Rehabil 2008;89:1230-1236.

Cameron MH. Physical agents in rehabilitation: from research to practice. 4th ed. Philadelphia (PA): Saunders; 2012.

Enoka RM. Activation order of motor axons in electrically evoked contractions. Muscle Nerve 2002;25:763-764.

Filipovic A, Kleinöder H, Dörmann U, Mester J. Electromyostimulation--a systematic review of the influence of training regimens and stimulation parameters on effectiveness in electromyostimulation training of selected strength parameters. J Strength Cond Res 2011;25:3218-3238. 
Finsterer J, Stöllberger C. Severe rhabdomyolysis after MIHA-bodytec electrostimulation with previous mild hyper-CK-emia and noncompaction. Int J Cardiol 2015;180:100-102.

Gondin J, Cozzone PJ, Bendahan D. Is high-frequency neuromuscular electrical stimulation a suitable tool for muscle performance improvement in both healthy humans and athletes? Eur J Appl Physiol 2011; 111:2473-2487.

Gondin J, Guette M, Ballay Y, Martin A. Electromyostimulation training effects on neural drive and muscle architecture. Med Sci Sports Exerc 2005;37:1291-1299.

Gregory CM, Bickel CS. Recruitment patterns in human skeletal muscle during electrical stimulation. Phys Ther 2005;85:358-364.

Harvey JR, Bradley MB, Aronica MJ. Psychological effects of functional electrical stimulation: a review of the literature. Neurorehabil Neural Repair 1992;6:203-211.

Hortobágyi T, Maffiuletti NA. Neural adaptations to electrical stimulation strength training. Eur J Appl Physiol 2011;111:2439-2449.

Kästner A, Braun M, Meyer T. Two cases of rhabdomyolysis after training with electromyostimulation by 2 young male professional soccer players. Clin J Sport Med 2015;25:e71-e73.

Kemmler W, Froehlich M, von Stengel S, Kleinoder H. Whole-body electromyostimulation - The need for common sense! Rationale and guideline for a safe and effective training. Dtsch Z Sportmed 2016b; 67:218-221.

Kemmler W, Schliffka R, Mayhew JL, von Stengel S. Effects of wholebody electromyostimulation on resting metabolic rate, body composition, and maximum strength in postmenopausal women: the Training and ElectroStimulation Trial. J Strength Cond Res 2010;24:1880-1887.

Kemmler W, Teschler M, Weißenfels A, Bebenek M, Fröhlich M, Kohl M, von Stengel S. Effects of whole-body electromyostimulation versus high-intensity resistance exercise on body composition and strength: a randomized controlled study. Evid Based Complement Alternat Med 2016a;2016:9236809.

Kemmler W, von Stengel S. Whole-body electromyostimulation as a means to impact muscle mass and abdominal body fat in lean, sedentary, older female adults: subanalysis of the TEST-III trial. Clin Interv Aging 2013;8:1353-1364.

Koch AJ, Pereira R, Machado M. The creatine kinase response to resistance exercise. J Musculoskelet Neuronal Interact 2014;14:68-77.

Lam H, Qin YX. The effects of frequency-dependent dynamic muscle stimulation on inhibition of trabecular bone loss in a disuse model. Bone 2008:43:1093-1100.

Lexell J, Taylor CC, Sjöström M. What is the cause of the ageing atrophy? Total number, size and proportion of different fiber types studied in whole vastus lateralis muscle from 15- to 83-year-old men. J Neurol Sci 1988;84:275-294.

Malnick SD, Band Y, Alin P, Maffiuletti NA. It's time to regulate the use of whole body electrical stimulation. BMJ 2016;352:11693.

Porcari JP, McLean KP, Foster C, Kernozek T, Crenshaw B, Swenson C. Effects of electrical muscle stimulation on body composition, muscle strength, and physical appearance. J Strength Cond Res 2002;16:165172.

Shankar K, Mihalko-Ward R, Rodell DE, Bucher A, Maloney FP. Methodologic and compliance issues in postcoronary bypass surgery subjects. Arch Phys Med Rehabil 1990;71:1074-1077.

Taylor AH, Fox KR. Effectiveness of a primary care exercise referral intervention for changing physical self-perceptions over 9 months. Health Psychol 2005;24:11-21.

von Stengel S, Bebenek M, Engelke K, Kemmler W. Whole-Body Electromyostimulation to Fight Osteopenia in Elderly Females: The Randomized Controlled Training and Electrostimulation Trial (TEST-III). J Osteoporos 2015;2015:643520. 